\title{
Decreased Uncorrected Visual Acuity
}

National Cancer Institute

\section{Source}

National Cancer Institute. Decreased Uncorrected Visual Acuity. NCI Thesaurus. Code C118716.

Diminished clarity of vision without optical correction (glasses and/or contacts). 